University of Wollongong

Research Online

Australian Institute for Innovative Materials -

Papers

Australian Institute for Innovative Materials

$1-1-2013$

Variation of pinning mechanism and enhancement of critical current density in MgB2 bulk containing self-generated coherent MgB4 impurity

Qi Cai

Tianjin University, caiqi6406608@163.com

Yongchang Liu

Tianjin University

Zongqing $\mathrm{Ma}$

Tianjin University, zqma@uow.edu.au

Huijun Li

Tianjin University

Liming Yu

Tianjin University

Follow this and additional works at: https://ro.uow.edu.au/aiimpapers

Part of the Engineering Commons, and the Physical Sciences and Mathematics Commons

Research Online is the open access institutional repository for the University of Wollongong. For further information contact the UOW Library: research-pubs@uow.edu.au 


\title{
Variation of pinning mechanism and enhancement of critical current density in MgB2 bulk containing self-generated coherent MgB4 impurity
}

\author{
Abstract \\ Bulk MgB2, with self-generated MgB4 pinning centers, have experienced two-step sintering process, \\ initially at $750^{\circ} \mathrm{C}$ and then $900-1000^{\circ} \mathrm{C}$. On the contrary to the widely accepted point that $\mathrm{MgB} 4$ \\ deteriorates superconductivity, it was found that MgB4 played a significant role in enhancing critical \\ current density. The precipitation pattern of MgB4 was studied from the lattice scale images. It was \\ observed that the initial coherent relation between the MgB 4 and the matrix was destroyed to become \\ semi-coherent and even incoherent as the second-step sintering temperature increased. Owing to the \\ lattice distortion caused by the elastic accommodation of the coherent interface, the small-sized MgB4 \\ particles controlled by the sintering temperature, and the fine grain connectivity affected by the porosity, \\ the critical current density was improved over the entire magnetic field. Finally, the dominating pinning \\ mechanism within the crystal was confirmed to be $\Delta K$ pinning in the two-step sintered MgB2 sample, \\ where the $K$ is the Ginzburg-Landau parameter, while the mechanism of one-step sintered sample is \\ surface pinning. 2013 AIP Publishing LLC.
}

\section{Keywords}

mgb4, bulk, mgb2, density, current, containing, critical, impurity, enhancement, mechanism, pinning, variation, self, generated, coherent

\section{Disciplines}

Engineering | Physical Sciences and Mathematics

\section{Publication Details}

Cai, Q., Liu, Y., Ma, Z., Li, H. \& Yu, L. (2013). Variation of pinning mechanism and enhancement of critical current density in MgB2 bulk containing self-generated coherent MgB4 impurity. Applied Physics Letters, 103 (13), 132601-1-132601-4. 


\section{AIP Aponed Phy is Letters}

Variation of pinning mechanism and enhancement of critical current density in MgB2 bulk containing self-generated coherent MgB4 impurity

Qi Cai, Yongchang Liu, Zongqing Ma, Huijun Li, and Liming Yu

Citation: Applied Physics Letters 103, 132601 (2013); doi: 10.1063/1.4822099

View online: http://dx.doi.org/10.1063/1.4822099

View Table of Contents: http://scitation.aip.org/content/aip/journal/apl/103/13?ver=pdfcov

Published by the AIP Publishing

\section{Articles you may be interested in}

Microstructural and crystallographic imperfections of MgB2 superconducting wire and their correlation with the critical current density

AIP Advances 4, 017113 (2014); 10.1063/1.4862670

Effect of processing temperature on high field critical current density and upper critical field of nanocarbon doped $\mathrm{Mg} B 2$

Appl. Phys. Lett. 90, 122502 (2007); 10.1063/1.2715026

High critical current density and improved flux pinning in bulk Mg B 2 synthesized by Ag addition J. Appl. Phys. 101, 043906 (2007); 10.1063/1.2655340

Critical Current Anisotropy, Pinning Properties and Relaxation Rate of "Ex-situ" MgB2/Fe Tapes AIP Conf. Proc. 824, 654 (2006); 10.1063/1.2192407

High critical current density of MgB 2 bulk superconductor doped with $\mathrm{Ti}$ and sintered at ambient pressure Appl. Phys. Lett. 79, 1154 (2001); 10.1063/1.1396629

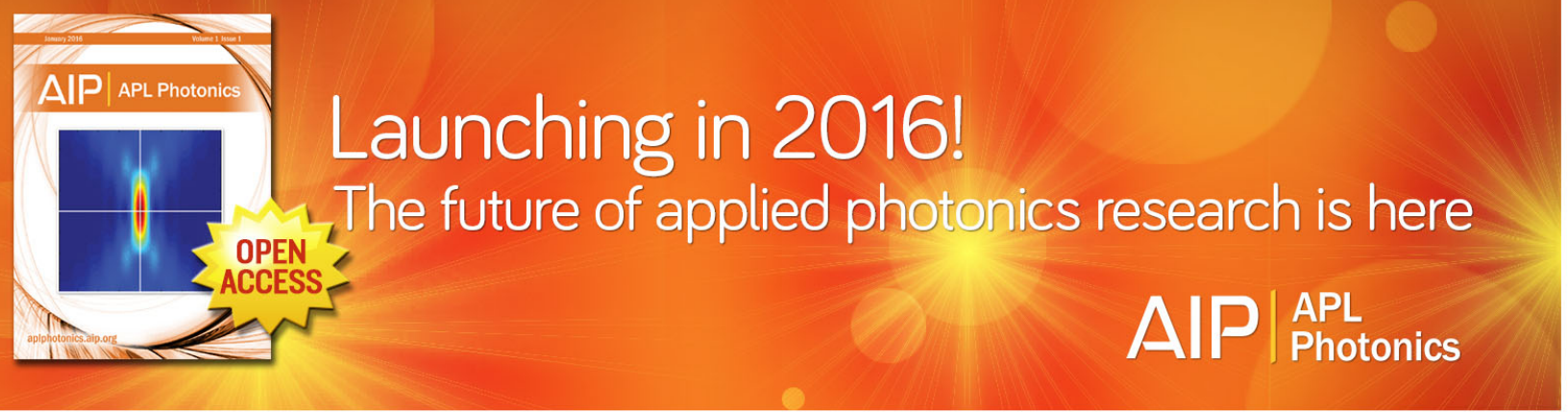




\title{
Variation of pinning mechanism and enhancement of critical current density in $\mathrm{MgB}_{2}$ bulk containing self-generated coherent $\mathrm{MgB}_{4}$ impurity
}

\author{
Qi Cai, Yongchang Liu, ${ }^{a}$ Zongqing Ma, Huijun Li, and Liming Yu \\ State Key Lab of Hydraulic Engineering Simulation and Safety, School of Materials Science \& Engineering, \\ Tianjin University, Tianjin 300072, People's Republic of China
}

(Received 10 July 2013; accepted 8 September 2013; published online 23 September 2013)

\begin{abstract}
Bulk $\mathrm{MgB}_{2}$, with self-generated $\mathrm{MgB}_{4}$ pinning centers, have experienced two-step sintering process, initially at $750{ }^{\circ} \mathrm{C}$ and then $900-1000{ }^{\circ} \mathrm{C}$. On the contrary to the widely accepted point that $\mathrm{MgB}_{4}$ deteriorates superconductivity, it was found that $\mathrm{MgB}_{4}$ played a significant role in enhancing critical current density. The precipitation pattern of $\mathrm{MgB}_{4}$ was studied from the lattice scale images. It was observed that the initial coherent relation between the $\mathrm{MgB}_{4}$ and the matrix was destroyed to become semi-coherent and even incoherent as the second-step sintering temperature increased. Owing to the lattice distortion caused by the elastic accommodation of the coherent interface, the small-sized $\mathrm{MgB}_{4}$ particles controlled by the sintering temperature, and the fine grain connectivity affected by the porosity, the critical current density was improved over the entire magnetic field. Finally, the dominating pinning mechanism within the crystal was confirmed to be $\Delta \kappa$ pinning in the two-step sintered $\mathrm{MgB}_{2}$ sample, where the $\kappa$ is the Ginzburg-Landau parameter, while the mechanism of one-step sintered sample is surface pinning. (C) 2013 AIP Publishing LLC. [http://dx.doi.org/10.1063/1.4822099]
\end{abstract}

The intermetallic compound $\mathrm{MgB}_{2}$ with the superconducting transition temperature of $39 \mathrm{~K}$ has been the focus of worldwide attention for the past ten years due primarily to its potential for practical magnetic and electrical applications. ${ }^{1}$ Although considerable progress have been made during this period, the application of pure $\mathrm{MgB}_{2}$ remains limited by the sensitivity of critical current density $\left(J_{\mathrm{c}}\right)$ to increasing applied magnetic field as a result of the relatively weak flux pinning. ${ }^{2}$

A series of exciting achievements in improving the $J_{\mathrm{c}}$ performance have been made by chemical doping method, which has been considered as the most convenient and effective way, owing to the dopant's talents in introducing extra electrons or second-phase particles., ${ }^{3,4}$ For example, carbon-containing compound addition, originating from $\mathrm{C}$ substitution for $\mathrm{B}$ sites in the lattice, would induce the lattice distortion and the devotion of one more electron, and thus enhance the $J_{\mathrm{c}}$. Elements $\mathrm{Cu}$ and $\mathrm{Y}$ addition would improve the $J_{\mathrm{c}}$ performance of $\mathrm{MgB}_{2}$ by forming nano-sized $\mathrm{MgCu}_{2}$ and $\mathrm{YB}_{4}$ second phases with $\mathrm{Mg}$ and $\mathrm{B}$, respectively. ${ }^{5-7}$ However, the dimension and the content of the second phase, even the sintering temperature and the original powder size, have to be seriously controlled, since only a small quantity of nano-scale particles embedded in the matrix were expected to function as effective pinning centers. As far as the investigation of pure $\mathrm{MgB}_{2}$, researchers have turned to mechanical milling to refine grains and irradiation to induce defects, both of which are likewise aimed at improving the pinning effects within the crystal. ${ }^{8,9}$ In view of that the operation of the applied techniques are generally complicated, it is meaningful to use a relatively easy way to introduce pinning centers into the $\mathrm{MgB}_{2}$ bulks.

\footnotetext{
${ }^{\text {a) }}$ Author to whom correspondence should be addressed. Electronic mail: licmtju@163.com. Tel./Fax: 0086-22-87401873.
}

When the sintering temperature is high and the $\mathrm{B}$ phase is locally excessive, $\mathrm{MgB}_{4}$ will generate as the impurity phase from $\mathrm{Mg}$ and $\mathrm{B}$ or $\mathrm{MgB}_{2}$ and $\mathrm{B} .{ }^{10}$ Little attention has been paid to the effects of $\mathrm{MgB}_{4}$ on the $J_{\mathrm{c}}$ performance of $\mathrm{MgB}_{2}$ in the past study. It was argued that the generation of $\mathrm{MgB}_{4}$ is the dominating reason that leads to the decrease of the critical current density. ${ }^{11}$ As we considered above, however, could the $\mathrm{MgB}_{4}$ serve as effective pinning centers if the particles were restricted to a small dimension and the content was controlled to be appropriate?

In this study, we returned to the investigation of pure $\mathrm{MgB}_{2}$. Two-step sintering method was employed, and a relatively high sintering temperature was set, with the purpose of self-generating $\mathrm{MgB}_{4}$ pinning centers. By restricting the content and the dimension, we expect to obtain $\mathrm{MgB}_{2}$ bulks with enhanced $J_{c}$ performance. In addition, we focused on the inherent mechanism of the enhancement of $J_{c}$, especially on the relationship between the precipitation pattern and the pinning mechanism.

Amorphous B powders (95\% purity, $100 \mathrm{~nm}$ in size) and Mg powders (99.5\% purity, $100 \mu \mathrm{m}$ in size) were mixed in an atomic ratio of $\mathrm{MgB}_{2}$. After ground thoroughly in an agate mortar, the mixture was pressed into cylindrical pellets $(\Phi 5 \times 1.5 \mathrm{~mm})$ under a pressure of $5 \mathrm{MPa}$. The obtained pellets were then sintered in the differential thermal analysis apparatus (Netzsch DSC 404C) at $750{ }^{\circ} \mathrm{C}$ for $0.5 \mathrm{~h}$ with a heating rate of $10 \mathrm{~K} \times \mathrm{min}^{-1}$, followed by heating up with the same rate to 900,950 , and $1000^{\circ} \mathrm{C}$, respectively. Without temperature holding, the samples were then cooled down to room temperature in the apparatus with a cooling rate of $40 \mathrm{~K} \times \min ^{-1}$. The whole process was accomplished under flowing high-purity $\mathrm{Ar}$ gas to prevent the samples from oxidation. The corresponding samples were designated as S750-900, S750-950, and S750-1000, respectively. Furthermore, the sample only sintered at $750{ }^{\circ} \mathrm{C}$ for $30 \mathrm{~min}$ 
was employed as a reference, and was denoted as S750. After that, the phase composition and microstructure were detected by means of X-ray diffraction (XRD, Rigaku $\mathrm{D} / \max 2500 \mathrm{CuK} \alpha$ ), scanning electron microscopy (SEM, Hitachi S-4800), and transmission electron microscopy (TEM, JEM-2100), respectively. The superconducting properties were measured by the magnetic property measuring system (SQUID-VSM, Quantum Design) after the samples were cut into a slab of size about $4 \times 2 \times 1 \mathrm{~mm}^{3}$. The corresponding $J_{\mathrm{c}}$ values were calculated from the width of magnetization hysteresis loops based on the extended Bean model: $J_{c}=20 \Delta M /[a /(1-a / 3 b)],{ }^{12}$ where $M$ is the volume magnetization, and $a$ and $b$ are the sample dimensions, orthogonal to $H$ (applied magnetic field).

Figure 1 shows the XRD patterns of the samples S750, S750-900, S750-950, and S750-1000. The sample only sintered at $750^{\circ} \mathrm{C}$ was composed of $\mathrm{MgB}_{2}$ as the main phase and $\mathrm{MgO}$ as the only inevitable impurity. In comparison, no additional characteristic peaks corresponding to new phase were distinguishable in the XRD pattern of sample S750-900. When the sample was further heated to $950{ }^{\circ} \mathrm{C}$ after holding at $750^{\circ} \mathrm{C}$, peaks of $\mathrm{MgB}_{4}$ phase, whose volume fraction was estimated to be $1.7 \%$ from the peak area in the XRD results, have been appeared. Furthermore, an increasing number of peaks indicating $\mathrm{MgB}_{4}$ phase was observed in the sample heated to $1000^{\circ} \mathrm{C}$, and the volume fraction of $\mathrm{MgB}_{4}$ was estimated to be $26 \%$, which is relatively excessive.

The lattice-scale HRTEM images of the samples S750-900, S750-950, and S750-1000 are shown in Fig. 2, from which the second phase $\mathrm{MgB}_{4}$ was found to embed in the matrix. The precipitation form of $\mathrm{MgB}_{4}$, especially the phase relation with the matrix, was explicit in the figure. Although no diffraction peaks of $\mathrm{MgB}_{4}$ exhibited in the XRD patterns, we found the $\mathrm{MgB}_{4}$ particles in the matrix of the sample S750-900 by matching the interplanar spacing with the Powder Diffraction File (PDF) card. More interestingly, a coherent interface was recognized between the $\mathrm{MgB}_{4}$ second-phase particle and the matrix, and a misfit accompanied by the lattice distortions, due to the elastic accommodation, also occurs both in the second-phase particles and the matrix. The inexistence of $\mathrm{MgB}_{4}$ peaks is

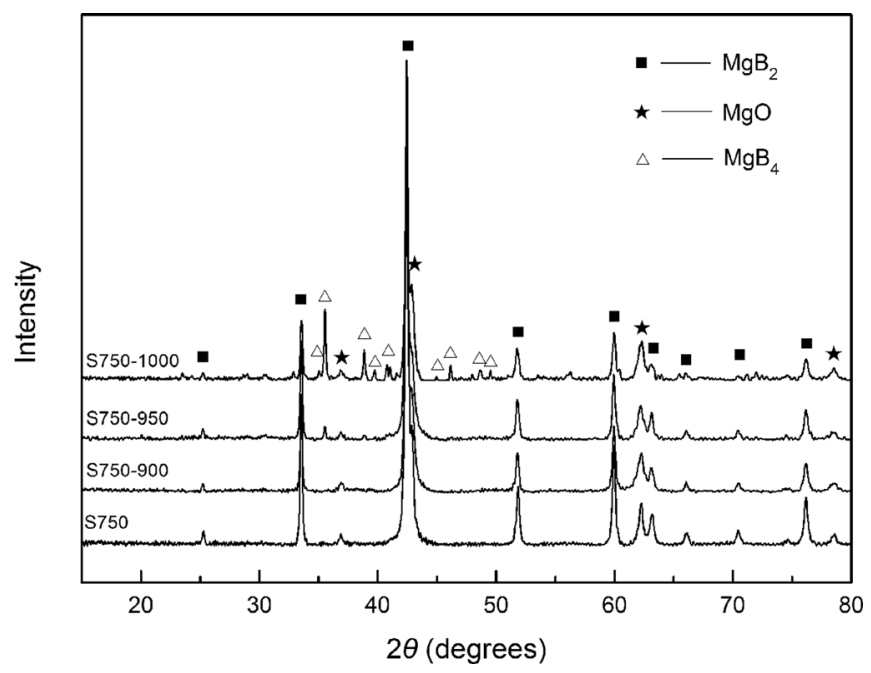

FIG. 1. X-ray diffraction patterns for one-step sintered $\mathrm{MgB}_{2}$ sample S750 and two-step sintered samples S750-900, S750-950, and S750-1000.
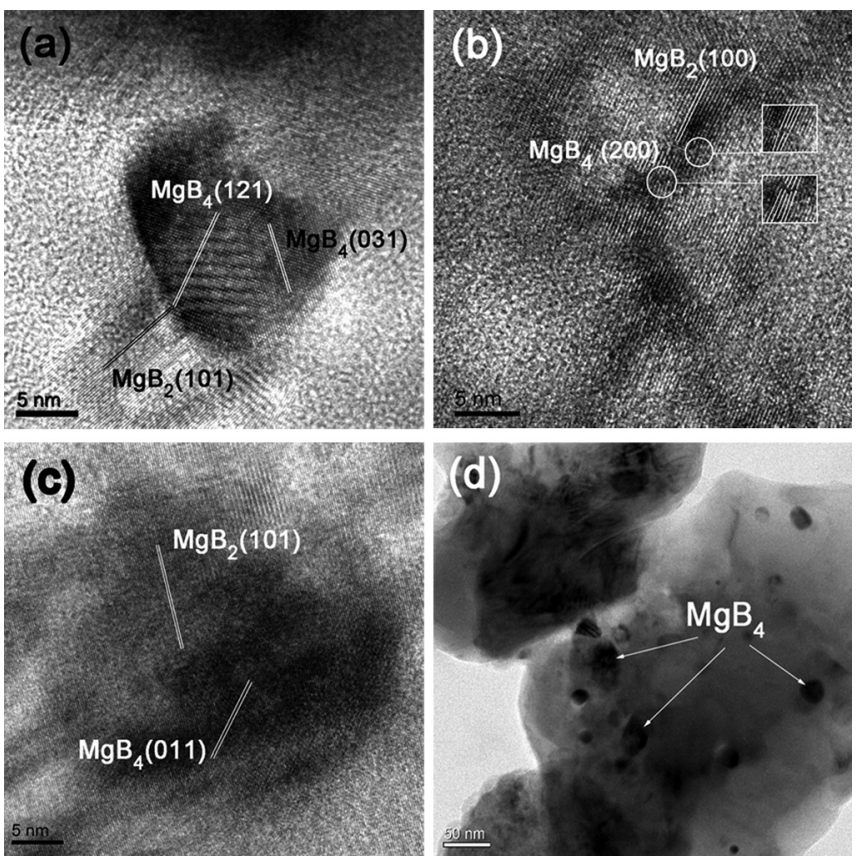

FIG. 2. Lattice scale HRTEM images for two-step sintered samples (a) S750-900, (b) S750-950, and (c) S750-1000, and (d) HRTEM image for sample S750-1000.

attributed to the coherency of diffraction caused by the coherent relation between the second phase and the matrix. ${ }^{13}$ When the sample was heated to a higher temperature, $950^{\circ} \mathrm{C}$, the coherent interface was partly destroyed, and misfit dislocations could be observed in Fig. 2(b). The relation between the second phase and the matrix became semicoherent, and edge dislocations with the extra half-planes oriented perpendicular to the interfacial plane. As a result, the lattice around the dislocations would become distorted. For sample S750-1000, the large amount of $\mathrm{MgB}_{4}$ (26 vol. \%) leads to its growth and aggregation, and the coherent relation was completely destroyed. The incoherent interface could be observed in Fig. 2(c).

On the other hand, the particle size of the second phase $\mathrm{MgB}_{4}$ in samples S750, S750-950, and S750-1000 was measured to be about 5,10 , and larger than $20 \mathrm{~nm}$, respectively. High temperature will lead to an increasing particle size of $\mathrm{MgB}_{4}$, even larger than the coherent length of $\mathrm{MgB}_{2}$, as in sample S750-1000 (see Fig. 2(d)). To sum up, small-sized $\mathrm{MgB}_{4}$ second phase generated at relatively low temperature $\left(<950^{\circ} \mathrm{C}\right)$ might serve as effective pinning centers, and the induced lattice distortion and dislocations due to the coherent or semi-coherent relation might pin the applied field as well. However, relatively high temperature $\left(>1000^{\circ} \mathrm{C}\right)$ would result in a large quantity of large particles (see Fig. 2(d)), which might be harmful to the critical current density.

The SEM images for samples S750, S750-900, S750950, and S750-1000 were shown in Fig. 3, from which the grain size and the porosities can be identified. Although the thermal process was changed, no apparent variation in grain size was observed in these samples. Considered the grains present cuboid, the average size (length of the cuboid) was measured to be about $800 \mathrm{~nm}$ in all the samples. Apart from this, it is noted that the difference between the samples lies in the porosity. The samples S750-950 and S750-1000 

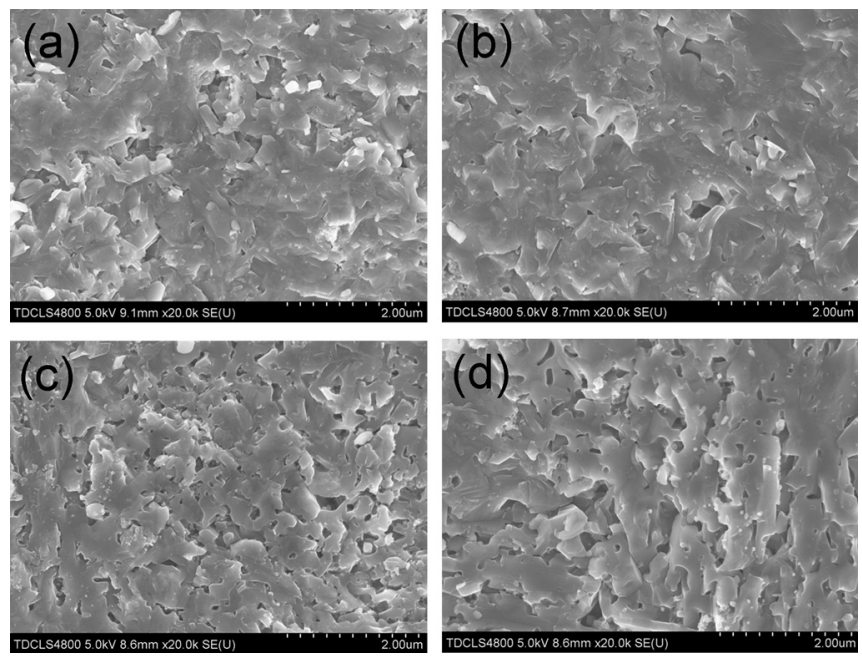

FIG. 3. SEM images for two-step sintered samples (a) S750, (b) S750-900, (c) S750-950, and (d) S750-1000.

contained more porous regions than the samples S750 and S750-900. Porosity is a key factor determining the critical current density by affecting the grain connectivity. The connectivity can be defined by a parameter $K, K=\Delta \rho_{g} / \Delta \rho$, where $\rho$ represents the resistivity, $\Delta \rho_{g}=6.32 \mu \Omega \times \mathrm{cm}$, and $\Delta \rho=\rho(300 \mathrm{~K})-\rho(40 \mathrm{~K}) .{ }^{14}$ The temperature dependence of resistivity for the samples was shown in Fig. 4(a), and using the resistivity values at $300 \mathrm{~K}$ and $40 \mathrm{~K}$, the $K$ values for the samples S750, S750-900, S750-950, and S750-1000 were calculated to be $0.215,0.408,0.251$, and 0.217 , respectively. The results indicated that the samples S750-900 and S750-950 showed better grain connectivity than the sample S750, and accordingly, the $J_{\mathrm{c}}$ performance is likely to be improved. However, the $K$ value for sample S750-1000 becomes decreased to almost the same level as sample S750, implying that the $J_{\mathrm{c}}$ values of sample S750-1000 may not be significantly affected by the variation of grain connectivity compared with sample S750, but by the large-sized secondphase particles.

The measured $J_{\mathrm{c}}-H$ characteristics of all the samples were illustrated in Fig. 4(b). As expected, the samples S750-900 and S750-950 exhibited excellent $J_{\mathrm{c}}$ performances. The highest $J_{\mathrm{c}}$ values were obtained in sample S750-900, which showed higher $J_{\mathrm{c}}$ values over the entire field in contrast with sample only sintered at $750^{\circ} \mathrm{C}$. However, sample S750-1000 showed uncompetitive current carrying capability. In order to obtain a deeper insight into the pinning properties, an extended analysis of $F_{\mathrm{p}}=\mu_{0} J_{\mathrm{c}} H$ is examined,

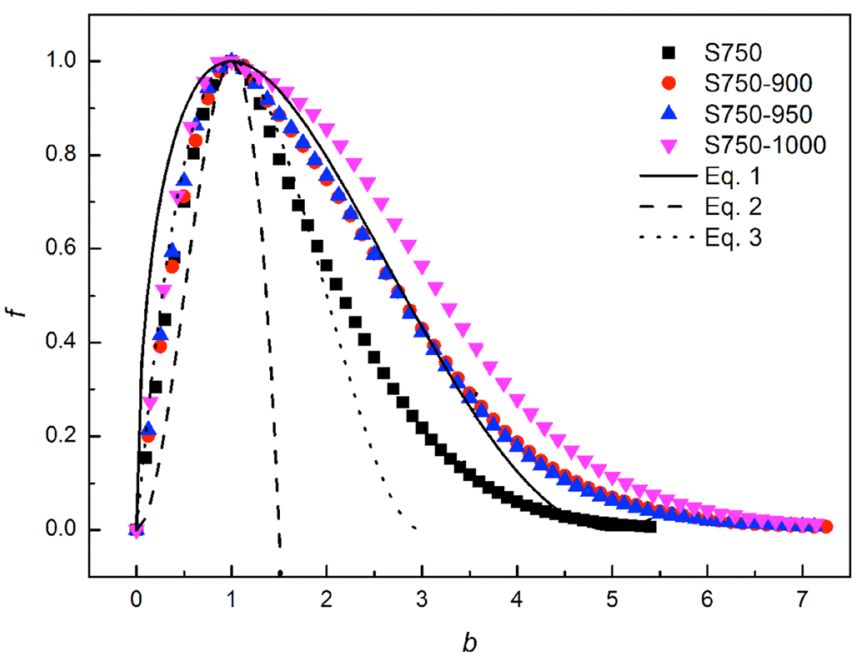

FIG. 5. Variation of $b\left(H / H_{\max }\right)$ dependence of flux pinning force $f\left(F_{\mathrm{p}} /\right.$ $\left.F_{\mathrm{p} \text {,max }}\right)$ and the fit curves for one-step sintered $\mathrm{MgB}_{2}$ sample $\mathrm{S} 750$, and twostep sintered samples S750-900, S750-950, and S750-1000.

where the $F_{\mathrm{p}}$ is the flux pinning force, and the $\mu_{0}$ is the magnetic permeability in vacuum. ${ }^{15}$ Normalized pinning force density $f=F_{\mathrm{p}} / F_{\mathrm{p}, \max }$ is often scaled with $b=H / H_{\text {max }}$, instead of $b=H / H_{\text {irr }},{ }^{16}$ and the normalized field $b$ dependence of the normalized flux pinning force $f$ was illustrated in Fig. 5. The scaling of $f-b$ for high- $T_{\mathrm{c}}$ superconductors is often analyzed by using the following equations: ${ }^{16-18}$

$$
\begin{gathered}
f(b)=3 b^{2}(1-2 b / 3), \\
f(b)=\frac{9}{4} b\left(1-\frac{b}{3}\right)^{2}, \\
f(b)=\frac{25}{16} \sqrt{b}\left(1-\frac{b}{5}\right)^{2} .
\end{gathered}
$$

The normalized force values of the three samples at low field are all in agreement with the fit curves of Eq. (2), indicating that below $H_{\max }$ the dominating mechanism is surface pinning. However, the values at high field became separated and are in accordance with different equations. The properties for sample S750 were dominantly affected by the surface pinning, since the grain boundary might be the only pinning effects in $\mathrm{MgB}_{2}$ sintered at $750^{\circ} \mathrm{C}$. For samples $\mathrm{S} 750-900$ and $\mathrm{S} 750-950$, the enhancement of the $J_{\mathrm{c}}$ performance should be attributed to the lattice distortion as well as the small content of $\mathrm{MgB}_{4}$ pinning centers, and the dominating mechanism is $\Delta \kappa$ pinning, whose fitting degree is the optimal
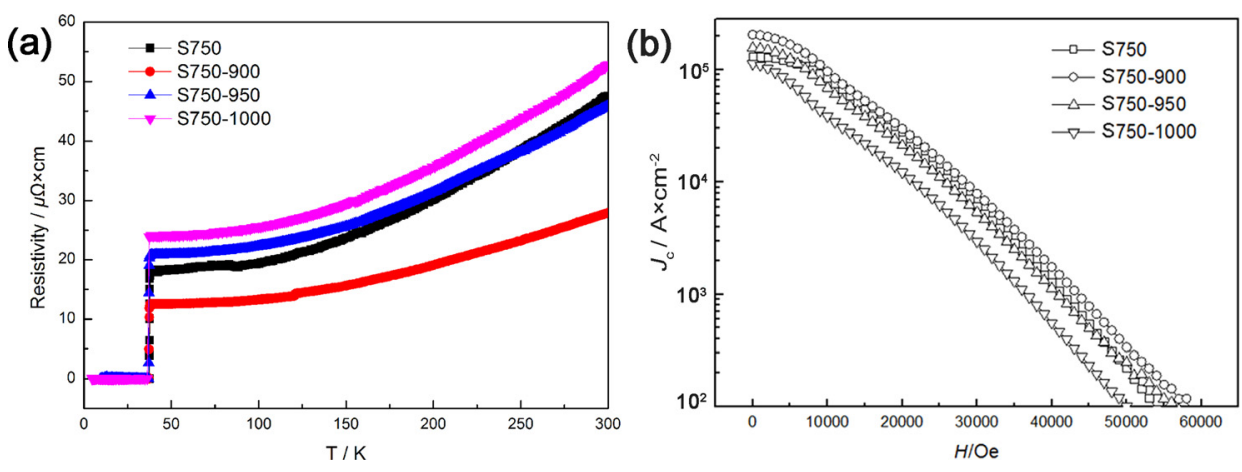

FIG. 4. (a) Temperature dependences of resistivity and (b) measured $J_{\mathrm{c}}-H$ characteristics at $20 \mathrm{~K}$ for one-step sintered $\mathrm{MgB}_{2}$ sample $\mathrm{S} 750$, and two-step sintered samples S750-900, S750-950, and $\mathrm{S} 750-1000$. 
in Fig. 5. The small $\mathrm{MgB}_{4}$ precipitates of $5-10 \mathrm{~nm}$ diameters contain $\mathrm{B}$ content slightly higher than that of the $\mathrm{MgB}_{2}$ matrix. Such compositional fluctuation will cause spatial variation of the Ginzburg-Landau parameter $(\kappa)$, which then provides additional flux pinning ( $\Delta \kappa$ pinning). ${ }^{19-21}$ Unfortunately, the pinning effects in sample S750-1000 was weakened as a result of the large particle size, as shown in Fig. 5, that the $F_{\mathrm{p}} / F_{\mathrm{p}, \max }$ values stay away from all the fit curves at high field.

The coherent or incoherent interface was largely associated with the precipitation process of $\mathrm{MgB}_{4}$, which may start with the formation of the particles. Very small local enrichments of B atoms first generated as soon as the $\mathrm{Mg}$ phase volatilized at the temperature higher than the melting point $649^{\circ} \mathrm{C}$, above which the standard Gibbs free energy $\left(\Delta \mathrm{G}^{\theta}\right)$ of the reaction $\mathrm{Mg}(\mathrm{l})+4 \mathrm{~B}(\mathrm{~s})=\mathrm{MgB} 4(\mathrm{~s})(-107.95$ $\left.+0.11 \mathrm{~T}, \mathrm{~kJ} \times \mathrm{mol}^{-1}\right)$ is more negative than that of the reaction $\mathrm{Mg}(\mathrm{l})+2 \mathrm{~B}(\mathrm{~s})=\mathrm{MgB}_{2}(\mathrm{~s}) \quad\left(-116.90+0.21 \mathrm{~T}, \quad \mathrm{~kJ} \times \mathrm{mol}^{-1}\right) .^{22}$ This indicates that the latter could proceed spontaneously and more easily than the former. The original motivation of sintering up to $900-1000^{\circ} \mathrm{C}$ followed by no holding process is to control the content of $\mathrm{MgB}_{4} \cdot \mathrm{MgB}_{4}$ first nucleates at the B-rich region with its (121) crystal face coherent with the (101) face of pre-generated $\mathrm{MgB}_{2}$. The $\mathrm{MgB}_{4}$ will further wrapped by the $\mathrm{MgB}_{2}$ that underwent a partial dissolution and re-precipitation after the $\mathrm{Mg}$ phase was melted. As the sintering temperature is relatively high, the $\mathrm{MgB}_{4}$ particles became coarse, and the coherent relation with the $\mathrm{MgB}_{2}$ matrix was destroyed, to semi-coherent relation, even incoherent relation.

In conclusion, the presence of the small-sized $\mathrm{MgB}_{4}$ impurities in bulk $\mathrm{MgB}_{2}$ prepared by two-step sintering has been observed to function as effective pinning centers and generate significant lattice distortion in the $\mathrm{MgB}_{2}$ lattice to yield improved $J_{\mathrm{c}}$ performance over the entire applied field. The fine grain connectivity also contributes to the improvement of $J_{\mathrm{c}}$ in sample experiencing two-step sintering at 750 and $900^{\circ} \mathrm{C}$. The lattice distortion mainly derives from the coherent or semi-coherent relation between $\mathrm{MgB}_{4}$ and the matrix, but the relation was destroyed to become incoherent when the second-step sintering temperature reaches $1000^{\circ} \mathrm{C}$, leading to a decrease in $J_{\mathrm{c}}$. Owing to the $\mathrm{MgB}_{4}$, the dominating pinning mechanism in $\mathrm{MgB}_{2}$ bulk varies from surface pinning to $\Delta \kappa$ pinning, which should be responsible for the enhancement in $J_{\mathrm{c}}$. Similar to the disappearance of coherent relation, however, the pinning effects become invalid eventually when the sintering temperature increased to $1000^{\circ} \mathrm{C}$, since the $\mathrm{MgB}_{4}$ has grown into large particles.

The authors are grateful to the National Natural Science Foundation of China (Grant Nos. 51077099 and 51302186) and the Natural Science Foundation of Tianjin (Grant No. 13JCZDJC32300) for grant and financial support.

${ }^{1}$ J. Nagamatsu, N. Nakagawa, T. Muranaka, Y. Zentani, and J. Akimitsu, Nature 410, 63 (2001).

${ }^{2}$ D. K. Finnemore, J. E. Ostenson, S. L. Bud'ko, G. Lapertot, and P. C. Canfield, Phys. Rev. Lett. 86, 2420 (2001).

${ }^{3}$ S. X. Dou, O. Shcherbakova, W. K. Yeoh, J. H. Kim, S. Soltanian, X. L. Wang, C. Senatore, R. Fukiger, M. Dhalle, O. Husnjak, and E. Babic, Phys. Rev. Lett. 98, 097002 (2007).

${ }^{4}$ A. Berenov, A. Serquis, X. Z. Zhao, Y. T. Zhu, D. E. Peterson, Y. Bugoslavsky, K. A. Yates, M. G. Blamire, L. F. Cohen, and J. L. MacManus-Driscoll, Supercond. Sci. Technol. 17, 1093 (2004).

${ }^{5}$ Y. Hishinuma, A. Kikuchi, Y. Iijima, Y. Yoshida, T. Takeuchi, and A. Nishimura, Supercond. Sci. Technol. 19, 1269 (2006).

${ }^{6}$ Z. Q. Ma, Y. C. Liu, Q. Z. Shi, Q. Zhao, and Z. M. Gao, J. Alloys Compd. 471, 105 (2009).

${ }^{7}$ J. Wang, Y. Bugoslavsky, A. Berenov, L. Cowey, A. D. Caplin, L. F. Cohen, J. L. M. Driscoll, L. D. Cooley, X. Song, and D. C. Larbalestier, Appl. Phys. Lett. 81, 2026 (2002).

${ }^{8}$ O. Perner, J. Eckert, W. Häßler, C. Fischer, K.-H. Müller, G. Fuchs, B. Holzapfel, and L. Schultz, Supercond. Sci. Technol. 17, 1148 (2004).

${ }^{9}$ M. Eisterer, M. Zehetmayer, S. Tonies, H. W. Weber, M. Kambara, N. H. Babu, D. A. Cardwell and L. R. Greenwood, Supercond. Sci. Technol. 15, L9 (2002).

${ }^{10}$ S. X. Dou, A. V. Pan, S. Zhou, M. Ionescu, H. K. Liu, and P. R. Munroe, Supercond. Sci. Technol. 15, 1587 (2002).

${ }^{11}$ C. E. J. Dancer, D. Prabhakaran, M. Basoğlu, E. Yanmaz, H. Yan, M. Reece, R. I. Todd, and C. R. M. Grovenor, Supercond. Sci. Technol. 22, 095003 (2009).

${ }^{12}$ C. P. Bean, Phys. Rev. Lett. 8, 250 (1962).

${ }^{13}$ N. E. V. Diaz, S. S. Hosmani, R. E. Schacherl, and E. J. Mittemeijer, Acta Mater. 56, 4137 (2008).

${ }^{14}$ A. Yamamoto, J. Shimoyama, K. Kishio, and T. Matsushita, Supercond. Sci. Technol. 20, 658 (2007).

${ }^{15}$ E. Martinez, P. Mikheenko, M. Martinez-lopez, A. Millan, A. Bevan, and J. S. Abell, Phys. Rev. B 75, 134515 (2007).

${ }^{16}$ T. Higuchi, S. I. Yoo, and M. Murakami, Phys. Rev. B 59, 1514 (1999).

${ }^{17}$ I. Shigeta, T. Abiru, K. Abe, A. Nishida, and Y. Matsumoto, Physica C 392-396, 359 (2003).

${ }^{18}$ Z. X. Shi, Y. X. Zhang, H. Lv, M. Xu, E.-M. Choi, and S.-I. Lee, Physica C 467, 101 (2007).

${ }^{19}$ W. A. Fietz and W. W. Webb, Phys. Rev. 178, 657 (1969).

${ }^{20}$ E. J. Kramer, J. Appl. Phys. 44, 1360 (1973).

${ }^{21}$ A. M. Campbell and J. E. Evetts, Adv. Phys. 21, 199 (1972).

${ }^{22}$ E. T. Turkdogan, Physical Chemistry of High Temperature Technology (Academic Press, New York, 1980). 